\title{
Alteração da camada de fibras nervosas da retina em usuários crônicos de cloroquina
}

\author{
Retinal nerve fiber layer alteration in chronic users of chloroquine
}

\author{
Daniela de Almeida Lyra Antunes ${ }^{1}$ \\ Hellmann Dantas Cavalcanti ${ }^{2}$ \\ Daena Leal ${ }^{3}$ \\ Helder Viana Santana ${ }^{4}$ \\ Rodrigo Almeida Vieira Santos ${ }^{5}$ \\ Laura Patrícia Ferreira Santos ${ }^{6}$
}

\begin{tabular}{|l|}
\hline RESUMO \\
\hline Objetivos: Avaliar a camada de fibras nervosas da retina (CFN) por meio da \\
polarimetria a laser, em pacientes em uso crônico de cloroquina. Métodos: \\
Foram estudados 44 olhos de 22 pacientes em uso de cloroquina por doenças \\
reumatológicas, por pelo menos um ano. Como controle, foram incluídos \\
vinte indivíduos sem uso de cloroquina com idade, gênero e raça similares. \\
Foram excluídos os pacientes que apresentavam história familiar de hiperten- \\
são ocular ou glaucoma. Ambos os olhos foram submetidos à análise da \\
camada de fibras nervosas da retina, com o aparelho GDx ${ }^{\circledR}$ Nerve Fiber \\
Analyser, pelo mesmo examinador. Resultados: Nos usuários crônicos de \\
cloroquina, verificou-se alteração em mais de dois parâmetros do GDx em 28 \\
olhos (63,6\%). Ocorreu também alteração no gráfico "Deviation from nor- \\
mal" com perda de fibras nervosas em 11 olhos (25\%). Quando comparado \\
com o grupo controle, os parâmetros que demonstraram diferença esta- \\
tisticamente significante foram: Superior Ratio, Inferior Ratio, Superior \\
Nasal, Elipse Modulation, The Number, Superior Average e Superior Inte- \\
gral. Houve também associação estatisticamente significante entre o tempo \\
de uso de cloroquina e perda da CFN. Conclusões: Comprovou-se a \\
associação entre o uso crônico da cloroquina e a alteração da CFN detectada \\
pelo GDx. Desta forma, esses resultados podem contribuir para o diagnóstico \\
precoce da perda de fibras nervosas na retinopatia por cloroquina.
\end{tabular}

Descritores: Cloroquina/efeitos adversos; Fibras nervosas/patologia; Retina/fisiopatologia; Doenças reumáticas/quimioterapia; Técnicas de diagnóstico oftalmológico; Estudo de casos e controles
Trabalho realizado na Fundação Altino Ventura - FAV. Recife (PE).

${ }^{1}$ Residente em Oftalmologia da Fundação Altino Ventura - FAV. Recife (PE).

${ }^{2}$ Preceptor do Departamento de Glaucoma da FAV. Reci$\mathrm{fe}(\mathrm{PE})$.

${ }^{3}$ Preceptora do Departamento de Visão Subnormal da FAV. Recife (PE).

${ }^{4}$ Residente em Oftalmologia da FAV. Recife (PE).

${ }^{5}$ Residente em Oftalmologia da FAV. Recife (PE).

${ }^{6}$ Residente em Oftalmologia da FAV. Recife (PE).

Endereço para correspondência: Fundação Altino Ventura - Rua da Soledade, 170 - Boa Vista - Recife (PE) CEP 50070-040

E-mail: fav@fundacaoaltinoventura.org.br

Recebido para publicação em 24.09.2003

Versão revisada recebida em 04.08.2004

Aprovação em 03.01.2005

\section{INTRODUÇ̃̃̃O}

Apesar da cloroquina e a hidroxicloroquina serem drogas utilizadas no manejo de algumas doenças reumatológicas, elas são perigosas para a visão e devem ser utilizadas apenas quando necessário e em pacientes que se submetem a exames oculares periódicos ${ }^{(1-3)}$.

O mecanismo pelo qual elas produzem toxicidade é desconhecido. A cloroquina e a hidroxicloroquina se unem à melanina e, portanto, se concentram na úvea e no epitélio pigmentar da retina. Além disso, as células ganglionares da retina são as primeiras a serem afetadas morfológica e bioquimicamente pela administração de doses tóxicas ${ }^{(1)}$. Depósitos superficiais na córnea pela cloroquina estão normalmente associados a nenhum ou apenas poucos sintomas visuais ${ }^{(4-5)}$.

As alterações retinianas são relacionadas à dose total ingerida, com efeito cumulativo, aparecendo mais raramente em doses totais inferiores a $250 \mathrm{~g} \mathrm{e}$ doses diárias inferiores a $250 \mathrm{mg}^{(2,6)}$. Na sua ação na retina, a cloroquina parece ser duas ou três vezes mais tóxica do que a hidroxicloroquina ${ }^{(7)}$. 
Quando alterações visíveis oftalmoscopicamente ocorrem na retina, a baixa visual é irreversível e pode progredir mesmo depois de cessado o uso da medicação ${ }^{(8)}$. Desta forma, a detecção da retinopatia em estágio precoce é imperativa, porque a descontinuidade da droga pode impedir a progressão e levar a condições pré-tratamento ${ }^{(7-9)}$.

Os métodos para diagnosticar os estados precoces da retinopatia por cloroquina são controversos ${ }^{(1)}$. Também tem se tornado aparente que a retinopatia pode estar presente com acuidade visual, teste de visão de cores, reflexo foveal e aparência de fundo de olho normais ${ }^{(10)}$. Existe, então, a necessidade de um teste que possa alertar os pacientes e oftalmologistas da possibilidade de diagnosticar os estágios precoces da retinopatia. Testes como a perimetria computadorizada e angiografia fluoresceínica são muito demorados e difíceis de realizar nestes pacientes, muitos deles idosos e com constante dor ${ }^{(11)}$.

O objetivo deste estudo foi avaliar a camada de fibras nervosas da retina (CFN) através da polarimetria a laser em pacientes em uso crônico de cloroquina. Através disto, busca-se o diagnóstico da maculopatia em estágios iniciais na tentativa de impedir a progressão e irreversibilidade do quadro.

\section{MÉTODOS}

Foram avaliados pacientes portadores de doenças reumatológicas em uso de cloroquina por pelo menos um ano e como grupo controle foram incluídos indivíduos, sem uso de cloroquina, com idade, gênero e raça similares.

Os critérios de inclusão para os dois grupos foram: idade de pelo menos 18 anos, sem história de doença ocular exceto estrabismo e sem história familiar de hipertensão ocular ou glaucoma. Todos os pacientes foram submetidos a exame oftalmológico completo.

Os critérios de exclusão foram: erro refracional maior que 5DE ou 2DC, acuidade visual corrigida menor que 20/40, opacidades corneanas ou cristalinianas em eixo visual, pressão intra-ocular maior que $21 \mathrm{mmHg}$, discos ópticos anômalos ou escavação maior que 0,5 ou ainda assimetria entre os dois olhos maior que $0,2^{(12)}$.

Ambos os olhos dos pacientes foram submetidos à análise da camada de fibras nervosas da retina (CFN) com o aparelho $\mathrm{GDx}^{\circledR}$ Nerve Fiber Analyzer (GDx) pelo mesmo examinador. Foram obtidas três imagens de cada olho aceitas pelos critérios de aquisição do aparelho e, somente considerada para estudo, a imagem média destas. Os resultados foram expressos por suas médias e respectivos desvios padrão. Com relação à variável contínua, foi utilizado o teste "t" de Student para amostras não pareadas. Para avaliação da diferença entre frequiências, foi utilizado o teste Exato de Fisher. Foi aceito $\mathrm{p}<0,05$ para rejeição da hipótese de nulidade.

\section{RESULTADOS}

Foram analisados 44 olhos de 22 pacientes portadores de doenças reumatológicas (13 com artrite reumatóide e $9 \mathrm{com}$ lupus eritematoso sistêmico), em uso de cloroquina por pelo menos um ano. Vinte e um eram do sexo feminino e um do masculino, com idades variando entre 21 e 71 anos $(48,1 \pm 10,6)$ com mediana de 42 anos. Como grupo controle foram incluídos vinte indivíduos, sem uso de cloroquina, com idade, gênero e raça similares. A média do tempo de uso de cloroquina foi de 4,0 $\pm 2,4$ anos variando de 1,5 a 8,0 anos (mediana - 3,4 anos). Todos os pacientes estavam usando a mesma dose diária $(150 \mathrm{mg})$.

Verificou-se alteração em mais de dois parâmetros do GDx em 28 olhos (63,6\%), havendo alteração no gráfico "Deviation from normal" (DFN) com perda de fibras nervosas em 11 olhos $(25 \%)$. Destes, cinco mostraram redução significativa da camada de fibras nervosas (CFN) apenas superior, dois superior e temporal, dois apenas temporal, enquanto um apresentou redução superior e nasal e um difusa (Tabela 1).

Os parâmetros que mais se alteraram nestes pacientes foram: Superior Average (29,5\%); Superior Integral (29,5\%); Superior Ratio (27,3\%) e Superior/Nasal (27,3\%). Os parâmetros dos grupos estudo e controle e os que demonstraram diferença estatisticamente significante estão mostrados na tabela 2.

Houve uma associação estatisticamente significante entre o tempo médio de uso de cloroquina e o resultado do GDx. Pacientes que utilizaram cloroquina por mais de três anos apresentaram maior número de parâmetros alterados do que aqueles com menos de três anos de uso $(\mathrm{p}=0,03)$ (Tabela 3$)$.

\section{DISCUSSÃO}

A obtenção da imagem da camada de fibras nervosas da retina é conseguida pelo GDx a partir da birrefringência que os axônios das células ganglionares apresentam à luz polarizada. Esta, ao passar através da CFN da retina, sofre um retardo que é proporcional a sua espessura ${ }^{(12)}$.

Baseando-se no fato de que as células ganglionares da retina são as primeiras a serem afetadas através da administração de doses tóxicas de cloroquina ${ }^{(1)}$, surgiu a proposta de um teste de rápida execução e com capacidade de detectar a maculopatia numa fase reversível, quando a descontinuidade da droga ainda possa impedir a progressão e levar a condições pré-tratamento.

\begin{tabular}{lcc|}
\hline \multicolumn{3}{c}{ Tabela 1. Distribuição das localizações e freqüências da perda da } \\
CFN em usuários crônicos de cloroquina
\end{tabular}




\begin{tabular}{|c|c|c|c|c|c|}
\hline \multirow[b]{2}{*}{ Parâmetros } & \multicolumn{2}{|c|}{$\begin{array}{l}\text { Usuários de } \\
\text { cloroquina }\end{array}$} & \multicolumn{2}{|c|}{$\begin{array}{l}\text { Grupo } \\
\text { controle }\end{array}$} & \multirow[b]{2}{*}{ Valor $\mathbf{p}^{*}$} \\
\hline & Média & $\overline{D P}$ & Média & DP & \\
\hline Symetry & 0,88 & 0,10 & 0,92 & 0,085 & 0,053 \\
\hline Sup. Ratio & 1,84 & 0,40 & 2,20 & 0,60 & 0,0019 \\
\hline Inf. Ratio & 2,20 & 0,41 & 2,42 & 0,52 & 0,036 \\
\hline Sup/Nasal & 1,89 & 0,27 & 2,08 & 0,32 & 0,005 \\
\hline Max. modul & 1,38 & 0,41 & 1,51 & 0,37 & 0,136 \\
\hline Elipse modul & 2,58 & 0,78 & 2,95 & 0,82 & 0,039 \\
\hline The number & 19,42 & 9,31 & 15,60 & 7,41 & 0,044 \\
\hline Av. thickness & 65,16 & 12,08 & 67,60 & 14,28 & 0,405 \\
\hline Elip average & 68,92 & 12,48 & 71,90 & 13,51 & 0,302 \\
\hline Sup. average & 71,40 & 14,19 & 77,55 & 11,54 & 0,035 \\
\hline Inf. average & 85,19 & 14,15 & 88,85 & 12,03 & 0,212 \\
\hline Sup. integral & 0,19 & 0,03 & 0,21 & 0,02 & 0,001 \\
\hline Teste $t$ de Studer & & & & & \\
\hline
\end{tabular}

Tabela 3. Distribuição das freqüências do tempo de uso de cloroquina e dos parâmetros alterados do GDx nos 22 pacientes estudados № de parâmetros alterados

Tempo de uso de cloroquina

$\leq 3$ anos

$>3$ anos $<2$ parâmetros $\geq 2$ parâmetros

Teste Exato de Fisher

Sabe-se que quando precocemente percebida e a droga imediatamente suspensa, a queda visual costuma estacionar, podendo em alguns casos desaparecer. Porém alguns pacientes continuam a piorar da visão, acreditando-se que isto ocorra pela liberação lenta de cloroquina armazenada nos tecidos hepáticos ${ }^{(2)}$.

Doses cumulativas de cloroquina acima de 250 g estão associadas à alteração funcional de células ganglionares da retina ${ }^{(2,6)}$. Todo paciente em uso de cloroquina deve ser periodicamente avaliado pelo oftalmologista, levando-se em consideração a dose diária e dose total ingerida. Também deve ser bem orientado a continuar regressando para controle, pelo risco crescente que a medicação lhe impõe.

Observou-se, neste trabalho, alteração estatisticamente significante na espessura da camada de fibras nervosas da retina em pacientes usuários de cloroquina. Porém estudos com maior amostra e mais tempo de observação desses indivíduos são necessários para confirmar cientificamente que esses achados são preditivos de maculapatia precoce.

\section{ABSTRACT}

Purposes: To evaluate the retina nerve fiber layer by laser polarimetry in patients in chronic use of chloroquine. Me- thods: Forty-four eyes of twenty-two patients were studied. These were in use of chloroquine due to rheumatic diseases during at least one year. As a control group, twenty patients without use of chloroquine with similar characteristics (age, gender and race) were included. Patients who had a family history of ocular hypertension or glaucoma were not included in this group. Both eyes were submitted to analysis of the retina nerve fiber layer with a GDx Nerve Fiber Analyzer. Results: In 28 eyes (63.6\%) of chronic chloroquine users, alterations in more than two parameters by GDx were found. There was alteration in the Deviation from the normal graph with a loss of nerve fibers in 11 eyes $(25 \%)$. When these results were compared with the control group, there was a significant statistical difference between the following parameters: Superior Ratio, Inferior Ratio, Superior Nasal, Elipse Modulation, The Number, Superior Average and Superior Integral. There was also a significant statistical association between the time of chloroquine use and loss of nerve fiber layer. Conclusions: From the results the following conclusion can be drawn: the chronic use of chloroquine was associated with alteration of the nerve fiber layer. Thus these results may contribute to early diagnosis of nerve fiber layer loss in chloroquine retinopathy.

Keywords: Chloroquine/adverse effects; Nerve fibers/pathology; Retina/physiopathology; Rheumatic diseases/drug therapy; Diagnostic techniques, opthalmological; Case-control studies

\section{REFERÊNCIAS}

1. Rodriguez FJ. Toxicidad retiniana y del epitelio pigmentario de la retina por cloroquina e hidroxicloroquina. Rev Colomb Reumatol. 2000;7(1):47-51.

2. Cruz MTP, Belfort Junior R. Doenças reumáticas. In: Belfort Junior R, Bonomo PP. Oftalmologia e clínica médica. São Paulo: Roca; 1983. p.58-9.

3. Bishara SA, Matamoros N. Evaluation of several tests in screening for chloroquine maculopathy. Eye. 1989;3(Pt 6):777-82.

4. Wetterholm DH, Winter FC. Histopathology of chloroquine retinal toxicity. Arch Ophthalmol. 1964;71:82-7.

5. Bartel PR, Roux P, Robinson E, Anderson IF, Brighton SW, van der Hoven HJ, Becker PJ. Visual function and long term chloroquine treatment. S Afr Med J. 1994;84(1):32-4.

6. Easterbrook M. Dose relationships in patients with early chloroquine retinopathy. J Rheumatol. 1987;14(3):472-5.

7. Ruiz RS, Saatci OA. Chloroquine and hydroxychloroquine retinopathy: how to follow affected patients. Ann Ophthalmol. 1991;23(8):290-1. Review.

8. Hart WM Jr, Burde RM, Johnston GP, Drews RC. Static perimetry in chloroquine retinopathy. Perifoveal patterns of visual fied depression. Arch Ophthalmol. 1984;102(3):377-80.

9. Mazzuca SA, Yung R, Brandt KD, Yee RD, Katz BP. Current practices for monitoring ocular toxicity related to hydroxychloroquine (Plaquetil) therapy. J Rheumatol. 1994;21(1):59-63.

10. Ehrenfeld M, Nesher R, Merin S. Delayed-onset chloroquine retinopathy. $\mathrm{Br}$ J Ophthalmol. 1986;70(4):281-3.

11. Easterbrook M. The use of Amsler grids in early chloroquine retinopathy. Ophthalmology. 1984;91(11):1368-72.

12. Giampani Junior J, Leal BC, Susanna Junior R. Analisador de fibras nervosas: um estudo sobre os resultados falso-positivos. Arq Bras Oftalmol. 2001;64 (1):9-12. 